\title{
Correction to: Characterization of the first fully human anti-TEM1 scFv in models of solid tumor imaging and immunotoxin-based therapy
}

\author{
Xiaopeng Yuan ${ }^{1,2} \cdot$ Mingjuan Yang $^{1} \cdot$ Xiang Chen $^{1} \cdot$ Xuhua Zhang $^{1,3} \cdot$ Shrey Sukhadia ${ }^{4} \cdot$ Najia Musolino $^{5} \cdot$ \\ Huijing Bao ${ }^{1}$. Tingtao Chen ${ }^{1}$. Chen $\mathrm{Xu}^{1}$. Qirui Wang ${ }^{1}$. Stephen Santoro ${ }^{1}$. Daniel Ricklin ${ }^{6}$. Jia Hu ${ }^{1} \cdot$ Ruihe Lin $^{1}$. \\ Wei Yang ${ }^{1} \cdot$ Zhijun Li $^{4} \cdot$ Weijun Qin $^{7} \cdot$ Aizhi Zhao ${ }^{1} \cdot$ Nathalie Scholler ${ }^{1,8} \cdot$ George Coukos $^{1,5}$
}

Published online: 8 January 2018

(c) Springer-Verlag GmbH Germany, part of Springer Nature 2018

\begin{abstract}
Tumor endothelial marker 1 (TEM1) has been identified as a novel surface marker upregulated on the blood vessels and stroma in many solid tumors. We previously isolated a novel single-chain variable fragment (scFv) 78 against TEM1 from a yeast display scFv library. Here we evaluated the potential applications of scFv78 as a tool for tumor molecular imaging, immunotoxin-based therapy and nanotherapy. Epitope mapping, three-dimensional (3D) structure docking and affinity measurements indicated that scFv78 could bind to both human and murine TEM1, with equivalent affinity, at a well-conserved conformational epitope. The rapid internalization of scFv78 and scFv78-labeled nanoparticles was triggered after specific TEM1 binding. The scFv78-saporin immunoconjugate also exerted dose-dependent cytotoxicity with high specificity to TEM1-positive cells in vitro. Finally, specific and sensitive tumor localization of scFv78 was confirmed with optical imaging in a mouse tumor model that has highly endogenous mTEM1 expression in the vasculature. Our data indicate that scFv78, the first fully human anti-TEM1 recombinant antibody, recognizes both human and mouse TEM1 and has unique and favorable features that are advantageous for the development of imaging probes or antibody-toxin conjugates for a large spectrum of human TEM1-positive solid tumors.
\end{abstract}

Keywords TEM1 $\cdot \mathrm{ScFv} \cdot \mathrm{Immunotoxin} \cdot$ Nanoparticle $\cdot$ Optical imaging

Abbreviations
3D $\quad$ Three-dimensional
aa $\quad$ Amino acid(s)

Xiaopeng Yuan, Mingjuan Yang, Xiang Chen and Xuhua Zhang contributed equally.

Co-supervision: Nathalie Scholler and George Coukos.

The original article can be found online at https://doi.org/10.1007/ s00262-016-1937-z.

Weijun Qin

qinweij@fmmu.edu.cn

$\triangle$ George Coukos

george.coukos@chuv.ch

1 Abramson Cancer Center, University of Pennsylvania, Philadelphia, PA, USA

2 Department of Laboratory Medicine, Zhujiang Hospital, Southern Medical University, Guangdong, China

3 School of Life Sciences, Zhengzhou University, Zhengzhou, Henan, China
ADC Antibody-drug conjugate(s)

ELISA Enzyme-linked immunosorbent assay

MOE Molecular operating environment

MRI Molecular magnetic resonance imaging

MTT 3-(4,5-Dimethylthiazol-2-yl)-2,5-Diphenyl Tetrazolium Bromide

NIR Near-infrared

NPs Nanoparticles

PBST PBS/Tween 20

PDB Protein data bank

4 Department of Chemistry and Biochemistry, University of the Sciences in Philadelphia, Philadelphia, PA, USA

5 Ludwig Institute for Cancer Research at CHUV, Rue du Bugnon 46-BH09-701, 1011 Lausanne, Switzerland

6 Department of Pathology and Laboratory Medicine, University of Pennsylvania, Philadelphia, PA, USA

7 Department of Urology, Xijing Hospital, Fourth Military University, Xi' an 710032, China

8 SRI International, Menlo Park, CA, USA 


\section{PET Positron emission tomography \\ RT Room temperature \\ RU Resonance unit \\ $\mathrm{scFv} \quad$ Single-chain variable fragment \\ SPR Surface plasmon resonance \\ TEM1 Tumor endothelial marker 1}

\section{Correction to: \\ Cancer Immunol Immunother (2017) 66(3):367-378 https://doi.org/10.1007/s00262-016-1937-z}

Due to errors in the original publication, the revised complete article is re-published here.

\section{Introduction}

Tumor cells are elusive targets for standard anti-cancer therapies due to their genetic heterogeneity and instability. Unlike tumor cells, tumor vasculature cells are genetically more stable, making them ideal for therapeutic targeting. The cells associated with the tumor vasculature, such as the vascular endothelium, pericytes, vasculature-associated monocytes and stroma cells provide critical support for tumor survival, growth, and invasion [1-3]. The elimination of these cells can produce substantial therapeutic results, in part because the tumor vasculature and stroma act as physical and molecular barriers that protect tumor cells from being targeted by the host immune system [4]. Macromolecules, such as antibodies, are often inefficient at penetration through obstacles such as high interstitial pressure and dense fibrosis [5,6]. In contrast to the tumor cells, endothelial cells are readily accessible directly via the bloodstream, enabling therapies that target the vasculature to avoid these pitfalls, and suggesting an explanation as to why the rate of response to therapeutic antibodies is much higher in leukemia than in solid tumors [7, 8]. Thus, vascular disruption mediated by therapeutic macromolecules has the potential to be a highly effective cancer therapeutic approach.

Endosialin, CD248, or TEM1, is an 80.9-kD surface protein expressed by tumor endothelial and endothelial progenitor cells [9], pericytes and tumor-associated fibroblasts [10-12]. TEM1 is found on the vasculature of many types of solid tumors [13-15] but is absent on normal vessels [13, 14]. TEM1 is implicated in vascular cell adhesion and migration, development [16, 17], neoangiogenesis [13, 14] and tumor progression [18]. TEM1 mRNA overexpression is associated with poor survival in breast cancer [19]. Importantly, $\mathrm{Tem}^{-/-}$mice present a striking reduction in tumor growth, invasiveness, and metastasis, but are otherwise healthy and exhibit normal wound healing [20]. Although the exact mechanism by which TEM1 promotes tumor invasion and metastasis is not very clear, which might be partially due to its interaction with matrix proteins [16, 17], it is conceivable that TEM1-targeted strategies may be highly effective in tumor imaging and therapy. In view of this, we therefore isolated a high affinity, fully human scFv78 from a yeast display scFv library [21]. In this study, we further characterized this scFv and evaluated its potential application in solid tumor targeted imaging and therapy.

\section{Materials and methods}

\section{Epitope mapping of scFv78 binding}

We isolated scFv78 in our previous study [21]. To localize the TEM1 epitope on which scFv78 binds, six fragments of approximately 70 amino acids (aa) with an overlap of 11-aa to cover all epitopes were amplified and co-transformed with linearized vector pAGA2 into yeast EBY100 to achieve display of the peptide T1 (20-88aa), T2 (78-152aa), T3 (142216aa), T4 (206-275aa), T5 (265-334aa), and T6 (324390aa) on the yeast surface. Then, domain epitope mapping was performed using these large peptides displayed on EBY100 yeast surface. Binding to the peptides was detected by flow cytometry as described previously [21, 22], and a series of 15-aa peptides with 10-aa overlap were synthesized to identify the potential linear epitope by competition enzyme linked immunosorbent assay (ELISA).

\section{Molecular modeling and scFv78-TEM1 docking}

Amino acid sequences of TEM1 and scFv78 were each taken as query sequences for similarity search against the Protein Data Bank (PDB) at http://www.pdb.org [23]. Similarity searches were performed using FASTA in an effort to identify homologous proteins of known structure for use as structure templates for model construction [24]. Structure-based sequence alignment between the template and the query sequence was generated using Molecular Operating Environment (MOE) software (Molecular Computing Group Inc. version 2011.10) with default parameters [25]. A 3D structural model of the query sequence was then created based on such alignment using the homology model tool in MOE. Docking of the 3D structural model of either human or murine TEM1 to the scFv78 structural model was performed using the protein-protein docking software ZDOCK (version 2.3) [26]. Hydrogen atoms were removed from both structures and the standard parameters from the uniCHARMM file were used for all atoms in both structures. ZDOCK searches all possible binding modes in the translational and rotational space between the two proteins and evaluates each position using an energy-based scoring function. The scoring function is composed of IFACE statistical 
potential, shape complementarity, and electrostatics. The docking complex with the best fit with the experimental data was reported.

\section{Affinity measurement of scFv78 to human TEM1 by surface plasmon resonance and ELISA}

The binding affinity of scFv78 to human TEM1 was determined by surface plasmon resonance (SPR) using a Biacore 3000 instrument (GE Healthcare) at $25^{\circ} \mathrm{C}$. Human TEM1 was biotinylated using the EZ-Link Sulfo-NHS-LC-Biotin reagent (Thermo Scientific, IL) at a protein:biotin molar ratio of 1:5. Biotinylated TEM1 was captured on a streptavidin-coated sensor chip (GE Healthcare) at a density of 1,000 resonance units (RU). A twofold dilution series of scFv78 $(1-500 \mathrm{nM})$ was injected at a flow rate of $20 \mu \mathrm{l} / \mathrm{min}$ and a contact time of $2 \mathrm{~min}$. After 5 min of dissociation, the TEM1 surface was regenerated using $2 \mathrm{M} \mathrm{NaCl}$. Binding signals were processed using Scrubber (v2; BioLogic, Campbell, Australia) by subtracting the responses of an empty streptavidin surface and of a series of buffer blanks. Kinetic analysis was performed over a concentration series of 1-8 nM using a 1:1 binding model in Scrubber to fit association rate $\left(k_{a}\right)$ and dissociation rate $\left(k_{d}\right)$ constants and calculate the binding affinity $\left(\mathrm{K}_{\mathrm{D}}=k_{d} / k_{a}\right)$.

Human and murine TEM1 were coated on Maxisorp plates (Nunc/Thermo Scientific, IL) overnight at $4{ }^{\circ} \mathrm{C}$ at concentrations of 0.8 and $0.4 \mu \mathrm{g} / \mathrm{ml}$, respectively. The next day, the plates were blocked with 5\% dry milk prepared in PBS/Tween 20 0.05\% (PBST). The plates were washed 6 times with PBST and incubated with 10-fold serially diluted scFv78 starting from $1 \mu \mathrm{M}$. After six washes with PBST, scFv78 binding was detected using 1:1000 diluted antiV5-HRP antibody (AbDserotec, NC). The binding curves were fitted using a non-linearized one-site binding model of GraphPad Prism 5 for the calculation of the affinities.

\section{Assessment of scFv78 internalization in cells in vitro}

The mouse endothelial cell line MS1 and the counterpart MS1-TEM1 that was transduced to express full-length human TEM1 (hTEM1) were seeded on microscope cover glass (Fisherbrand, Loughborough) in 24-well plates. The following day, the cover glasses were transferred into new plates for incubation with fresh medium containing $1 \mu \mathrm{M}$ scFv78 for 2, 6 and $15 \mathrm{~h}$. A non-relevant scFv was used as a negative control for binding [21]. After incubation with the proteins, the cells were fixed, permeabilized with $0.1 \%$ Triton and $0.2 \%$ BSA-PBS, and then blocked with $2 \%$ BSAPBS for $30 \mathrm{~min}$. Both $\mathrm{scFv} 78$ and the control $\mathrm{scFv}$ proteins have a V5 tag at the $\mathrm{C}$ terminus, and Alexa 647 conjugated anti-V5 antibody (AbDserotec, NC) was therefore used to detect their internalization. DAPI was added at a 1:4000 dilution to stain the nucleus. Finally, the cover glasses were mounted on slides for the assessment of internalization by confocal imaging.

\section{ScFv78-nanoparticle conjugate internalization by cells in vitro}

Streptavidin-coated iron oxide nanoparticles (NPs) (MiltenyiBiotec, CA) with a diameter of $50 \mathrm{~nm}$ were used for the specific internalization assay. scFv78 was site-specifically biotinylated in yeast to obtain scFv78-bio as described previously. BSA was labeled with biotin using the EZ-Link SulfoNHS-LC-Biotin reagent (Thermo Scientific, IL) [21, 27], and then half of the biotinylated BSA was further labeled by NHS-Dylight649 (Thermo Scientific, IL) to generate BSA-bio-Dylight 649 (Fig. 3a). ScFv78-bio and BSA-bioDylight 649 were mixed at a 1:1 molar ratio and incubated with streptavidin-coated NPs for $1 \mathrm{~h}$ at RT. BSA-bio and BSA-bio-Dylight 649 were also labeled with the NPs as a negative control (Fig. 3b). The labeled NPs were purified with a magnet, and dye labeling efficiency was assessed by flow cytometry. For internalization assays, the labeled NPs were washed twice with PBS and resuspended in complete DMEM medium; then, labeled NPs were added to MS1 or MS1-hTEM1 cells. After overnight culture, the cells were washed with PBS prior to nuclear staining with DAPI. Cells were fixed with $2 \%$ paraformaldehyde solution and NP internalization was evaluated by confocal imaging.

\section{TEM1-targeted toxin delivery by thescFv78 immunoconjugate in vitro}

ScFv78 was conjugated with the ribosome-inactivating protein saporin (ZAP) to evaluate whether scFv78 may be used as a vehicle for theTEM1-targeted delivery of toxins. Sitespecific biotinylated scFv78 (scFv78-bio) was conjugated with the streptavidin-labeled saporin (Advanced Targeting Systems, CA) by incubation at room temperature (RT) for $1 \mathrm{~h}$ at a molar ratio of 4:1 (scFv78:ZAP). Cells were cultured in 96-well plates to $30 \%$ confluence and then incubated for $96 \mathrm{~h}$ in the presence of 10-fold serially diluted ZAP, scFv78 or scFv78-ZAP starting from $40 \mathrm{nM}$ down to $0.04 \mathrm{nM}$. The viability of MS1 and MS1-hTEM1 cells was then determined by MTT colorimetric assay.

\section{In vivo TEM1-targeted optical imaging with scFv78}

Nude mice received subcutaneous inoculation of $5 \times 10^{5}$ $\mathrm{TC} 1$ cells on the bilateral hip. All animal experiments were under University of Pennsylvania Institutional Review Board Protocol 702679, and were conducted in compliance with Institutional Animal Care and Use Committee guidelines. Tumors were allowed to develop for 10 days, reaching an 
approximate size of $0.5 \mathrm{~cm}$ in diameter, prior to optical imaging. One milligram of scFv78 and the control scFv were labeled with a near-infrared (NIR) dye, IRDye800CW-NHS (LI-COR Biosciences, NE), by incubation of the respective protein with 20 -fold excess dye for $2 \mathrm{~h}$ at $4{ }^{\circ} \mathrm{C}$. Free dye was removed by dialysis against PBS. Two hundred micrograms of dye-labeled scFv78 and control scFv in $200 \mu \mathrm{l}$ PBS were injected into the mice via the tail vein, and their biodistributions were monitored by a real-time imaging system (Pearl Impulse Small Animal Imaging System) at $30 \mathrm{~min}, 1,3$, $6,9,24,48$ and $72 \mathrm{~h}$ post-injection. Mice were sacrificed after the final imaging. Signal intensities in the tumor, liver and kidneys in both groups were quantified using the Pearl Impulse Small Animal Imaging System software and their relative intensities were plotted against the background.

\section{TEM1 expression assessment in vivo}

The tumor tissue, liver and kidneys were collected and analyzed for TEM1 expression by real-time PCR. Total RNA was extracted from different tissues, as well as MS1 cells (mTEM1-negative), 2H11 cells (mTEM1-positive) and TC1 cells (with low mTEM1 expression). The probe for mTEM1 or GAPDH (Mm00547485_s1, Mm99999915_g1, Applied Biosystems/Invitrogen, NY) was mixed with cDNA and reaction buffer, which was then subjected to a $94{ }^{\circ} \mathrm{C}$ denaturation step for $10 \mathrm{~min}$, followed by 40 cycles of two-step amplification, a $94{ }^{\circ} \mathrm{C}$ denaturation step for $15 \mathrm{~s}$ and $60{ }^{\circ} \mathrm{C}$ annealing and extension steps for $1 \mathrm{~min}$. The relative expression levels of mTEM1 in different tissues were calculated using the MS1 cells as a reference.

\section{Results}

\section{ScFv78 binds to a conserved conformational epitope of mouse and human TEM1 in the extracellular domain}

Using a yeast display system, we successfully displayed each of six peptides derived from the 18-390 aa extracellular $\mathrm{N}^{\prime}$ terminal domain of hTEM1. Each peptide was engineered to have an 11-aa overlap with the adjacent peptide (Fig. 1a). In addition to the full-length protein (T1.2, 18-390 aa), flow cytometry data revealed that scFv78 was only able to bind peptide fragment six, T6 (324-390 aa) (Fig. 1b). To further characterize the binding site, a series of overlapping 15 -aa peptides were generated from the T6 fragment. In a competition assay, none of the 15 -aa peptides were able to disrupt the binding of scFv78 to the T6 fragment, even at 100-fold higher concentration (data not shown). This suggests that scFv78 binds to a conformational rather than a linear epitope. Furthermore, we were able to show that scFv78 can only bind to native TEM1 in ELISA and flow cytometry but not to denatured TEM1 in western blot (data not shown). Human TEM1 shares $87 \%$ amino acid sequence identity with its murine counterpart over the T6 region (324-390 aa) (Fig. 1f). Our previous data indicate that scFv78 also binds to endogenous murine TEM1 expressed on the $2 \mathrm{H} 11$ cell line [21]. Together, these findings suggest that scFv78 binds to a conserved conformational epitope, which is likely shared between the human and murine TEM1.

\section{Modeling of the scFv78-TEM1 complex confirms the conformational epitope in the T6 peptide}

Threading analysis using the T6 sequence from both the human and murine TEM1 identified a structural domain of potential homology within a region of the $\mathrm{OmpR} / \mathrm{PhoB}$ (124-225 aa) protein from thermotoga maritima (PDB ID: 1KGS). The identification was deemed reliable, as the $\mathrm{Z}$-score was above the threshold of 3.5. Consistent with the fact that the two TEM1 fragments have $87 \%$ sequence identity, this finding supports the hypothesis that the conformation of this T6 region in both species remains conserved. The 3D structure of the T6 region of both human and murine TEM1 sequence was thus modeled using this structural domain (Fig. 1f).

Through similarity search, the $\mathrm{B} / \mathrm{D}$ chain of a human antiIFN antibody (PDB code: 3UX9) was identified as sharing $63 \%$ sequence identity with scFv78, making it suitable as a comparative modeling template. The 3D structure of scFv78 was modeled based on the chain B structure of the anti-IFN antibody (Fig. 1d). The structural models of the T6 region of the human and murine TEM1 were then docked into the 3D model of the antibody fragment scFv78 (Fig. 1d). For the human or mouseTEM1-scFv78 complexes, the best docking models had ZDOCK scores of 57.13 and 53.24, respectively (Fig. 1d). In addition, both T6 structures fit well within the pocket of the CDR regions of scFv78, and several suggestive interactions between residues of the T6 structures and scFv78 were observed. One of these interactions occurred between 367 and 378 aa and the CDR loops in scFv78. Based on our docking models, the folding of the T6 sequence appears to be critical for the formation of multiple interactions between $\mathrm{T} 6$ and scFv78. Additionally, scFv78 recognizes the full-length $\mathrm{T} 6$ fragments but is not competed away by any of the derivative 15-aa peptides; these data support the assertion that scFv78 binds to a conformational but not the linear epitope of TEM1.

\section{ScFv78 binds to human and mouse TEM1 with the same high affinity}

The evaluation of the interaction between hTEM1 and scFv78 by SPR showed dose-dependent binding with 
a

hTEM1 extracellular $\mathrm{N}$ terminal peptide (20-390 aa)

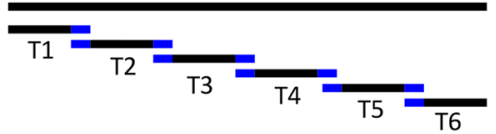

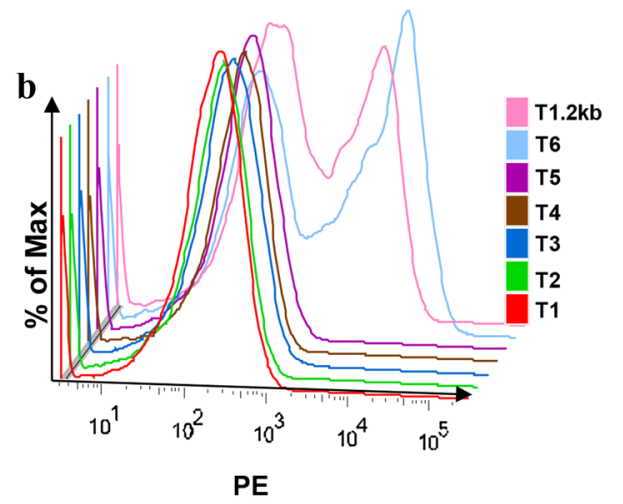

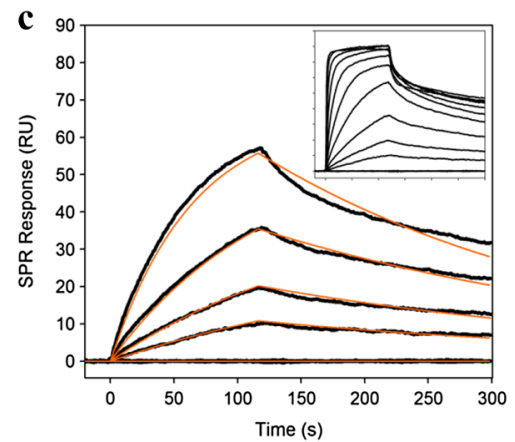

d
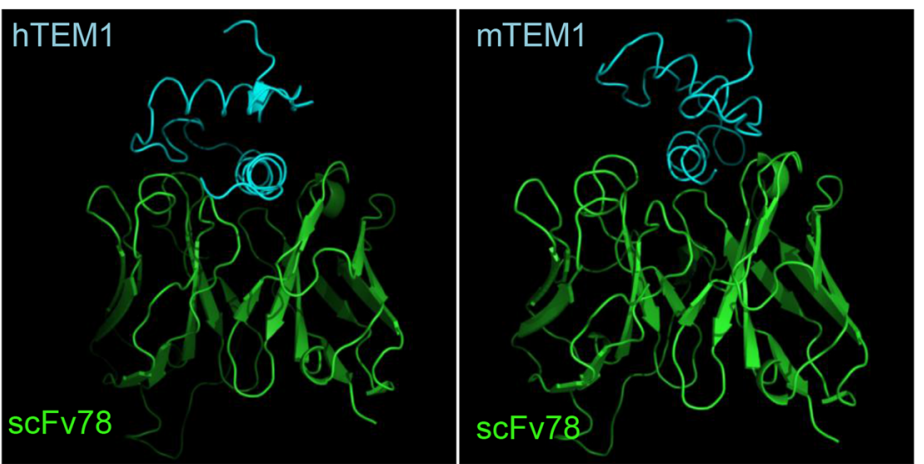

e Affinity measurement of ScFv78

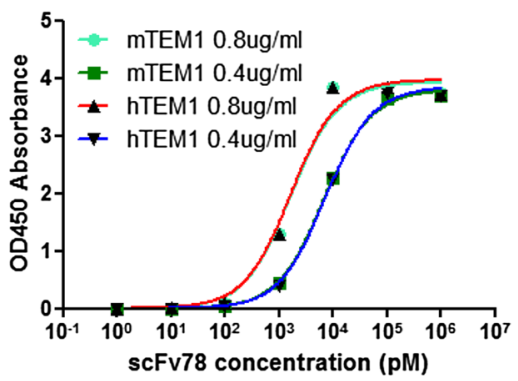

f

\begin{tabular}{|c|c|c|c|c|c|c|c|}
\hline \multicolumn{2}{|c|}{$\begin{array}{l}\text { Score } \\
99.0 \text { bits(245) }\end{array}$} & $\begin{array}{l}\text { Expect } \\
5 e-24()\end{array}$ & $\begin{array}{l}\text { Method } \\
\text { Composition-based stats. }\end{array}$ & $\begin{array}{l}\text { Identities } \\
58 / 67(87 \%)\end{array}$ & $\begin{array}{l}\text { Positives } \\
62 / 67(92 \%)\end{array}$ & $\begin{array}{ll}\text { Gaps } & \text { Frame } \\
0 / 67(0 \%) & \\
\end{array}$ & \\
\hline Query & 1 & \multicolumn{5}{|c|}{$\begin{array}{l}\text { QMCVNYVGGFECYCSEGHELEADGISCSPAGAMGAQASQDLGDELLDDGEDEEDEDEAWK } \\
\text { QMCVNYVGGFECYCSEGHELEADGISCSPAGAMGAQASQDL DELLDDGE+ EDE+E W+ }\end{array}$} & 60 \\
\hline Sbjct & 324 & \multicolumn{5}{|c|}{ QMCVNYVGGFECYCSEGHELEADGISCSPAGAMGAQASQDLRDELLDDGEEGEDEEEPWE } & 383 \\
\hline Query & 61 & $\begin{array}{l}\text { AFNGGWT } \\
\text { E+G WI }\end{array}$ & 67 & & & & \\
\hline Sbjct & 384 & DEDGTW & 390 & & & & \\
\hline
\end{tabular}

Fig. 1 Epitope mapping, affinity measurement and 3D docking of scFv78. a The 20-390 aa extracellular domain of hTEM1 was divided into six segments with 11-aa overlap (T1-T6). T1: 20-88 aa; T2: 78-152 aa; T3: 142-216 aa; T4: 206-275 aa; T5: 265-334 aa; T6: 324-390 aa. b Binding of biotinylated scFv78 to the peptides. T1.2 kb represents the control 20-390 aa peptide. c Affinity of scFv78 to human TEM1 measured by SPR. The binding signals (black) of scFv78 (1-500 nM) were evaluated to extract affinity and kinetic rate constants using a 1:1 binding model (red fit). A range of $1-8 \mathrm{nM}$ was used for the kinetic fit due to a slight heterogeneity effect observed at higher concentrations in the full dataset (insert). d Affinity of scFv78 to murine TEM1 measured by ELISA. e The threedimensional structural models of the T6 region of human (e, left) and murine (e, right). Cyan, the 324-390 aa domain of TEM1; green, represents antibody fragment scFv78. f Murine and human TEM1 sequence alignment (324-390 aa) an affinity $\left(\mathrm{K}_{\mathrm{D}}\right)$ of $2 \mathrm{nM}$ and a kinetic fit to a 1:1 model $\left(k_{a}=1 \times 10^{6} \mathrm{M}, k_{d}=3 \times 10^{-3} \mathrm{M}\right)($ Fig. $1 \mathrm{c})$. The interaction was saturable, yet deviations from the $1: 1$ model were observed at a higher concentration range (Fig. 1c, insert), most likely due to heterogeneities during the biotinylation of hTEM1. The binding affinity of scFv78 to mTEM1 was measured by ELISA and compared with that of the human protein. scFv78 was found to bind both proteins with exactly the same low nanomolar affinity of $1.6 \mathrm{nM}$ (Fig. 1e).

\section{ScFv78 is rapidly internalized specifically in TEM1-expressing cells}

Confocal microscopy showed significant and specific internalization of scFv78 into the MS1-hTEM1 cells but not MS1 cells, while untreated cells or cells treated with a control protein showed no luminescence (Fig. 2). Incubation periods longer than $2 \mathrm{~h}$ did not enhance scFv78 internalization in MS1-hTEM1 cells, indicating a rapid internalization of the antibody fragment. The internalized $\mathrm{scFv} 78$ protein 
Fig. 2 TEM1-specific internalization mediated by scFv78. a-f MS1 (TEM1-)cells were incubated with scFv78 and control $\mathrm{scFv}$ in complete medium at $37^{\circ} \mathrm{C}$ for 2 , 6, or 15 h. g-l MS1TEM1 (TEM1+) cells were incubated with scFv78 and control $\mathrm{scFv}$ in complete medium at $37^{\circ} \mathrm{C}$ for 2,6 , or $15 \mathrm{~h}$

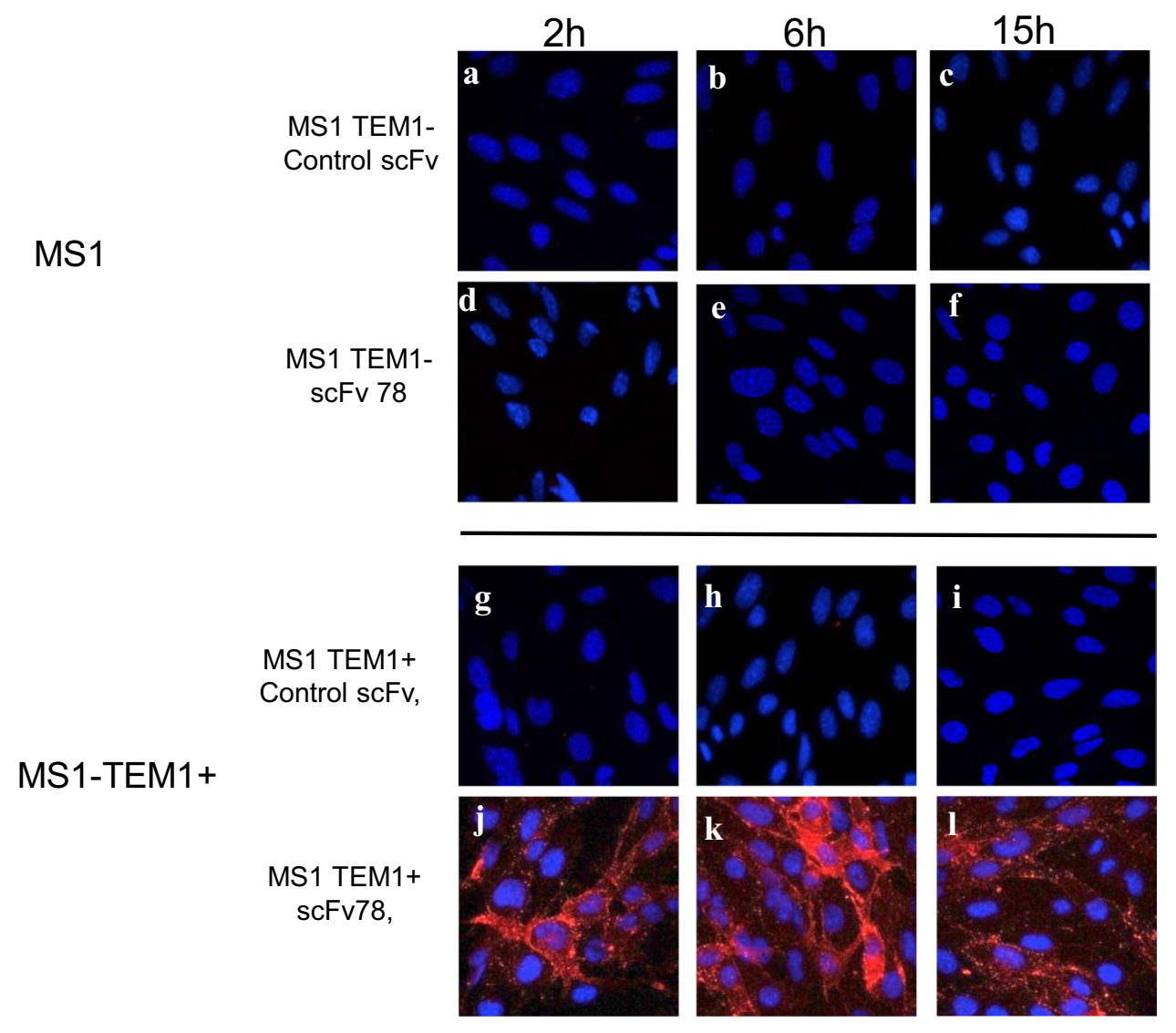

appeared as evenly distributed dots in the cytoplasm, suggesting localization in the endosome/lysosome compartment.

\section{ScFv78 mediates NP-specific internalization in TEM1-expressing cells}

To test the ability of scFv78 to mediate NP internalization, we prepared iron oxide NPs conjugated with scFv78 and labeled with Dylight 649 (Fig. 3a, b). Analysis indicated that the scFv78-conjugated NPs and the control BSA-conjugated NPs had similar fluorescence intensity (Fig. 3c). After incubation with cells overnight, only the scFv78-conjugated NPs showed significant internalization specifically into the hTEM1-positive cell line, while the BSA-labeled NPs showed no internalization in either cell line (Fig. 3d). The internalized NPs were mainly located around the nucleus. This indicates that $\mathrm{scFv} 78$ could mediate significant and specific internalization only in TEM1-positive cells.

\section{ScFv78 mediates the specific killing of TEM1-expressing cells with immunotoxin}

To test whether scFv78 could be used to deliver an immunotoxin selectively to TEM1- positive cells, we conjugated biotinylated scFv78 (scFv78-bio) with streptavidin-coupled saporin (scFv78-ZAP) and investigated the toxicity of the immunotoxin conjugate on human and mouse TEM1-positive cells. The results showed that the control toxin ZAP had no effect on either MS1 or MS1-hTEM1 cells (Fig. 4a), as it could not be internalized. In comparison, scFv78-ZAP showed dose-dependent cytotoxicity specifically when cultured with the MS1-TEM1 cells (Fig. 4b). Statistical significance was observed at the concentrations between $4 \mathrm{nM}$ $(\mathrm{p}<0.05)$ and $40 \mathrm{nM}(\mathrm{p}<0.01$, t-test $)$, and led to a dramatic growth arrest of the MS1-hTEM1 cells (Fig. 4b).

\section{ScFv78 allows for specific in vivo tumor imaging}

IRDye 800CW-labeled scFvs were tail-vein injected, and the in vivo real-time biodistribution was monitored using a LI-COR infra-red imaging system. The control conjugate was found to enrich mainly in the liver as most antibodies, probably as a result of capture by Kupffer cells (Fig. 5c, d) [28, 29]. In comparison, the scFv78-IRDy800CW conjugate demonstrated significant accumulation at the site of the tumor (Fig. 5e). This accumulation was seen as early as $30 \mathrm{~min}$ post-injection and lasted for least $48 \mathrm{~h}$ (data not shown) for scFv78, with the highest signal detected at $6 \mathrm{~h}$ post injection (Fig. 5a, b). Some accumulation was also observed in the kidneys of both groups, possibly due to the 
a

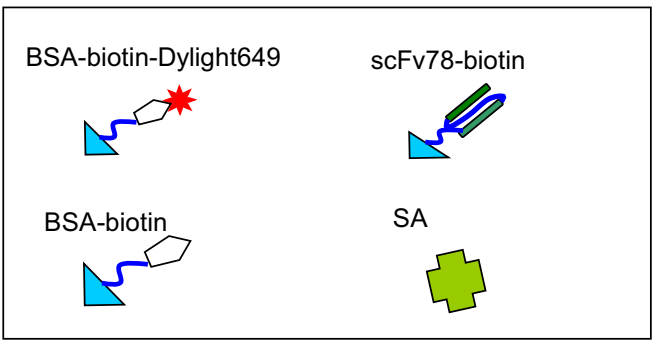

c

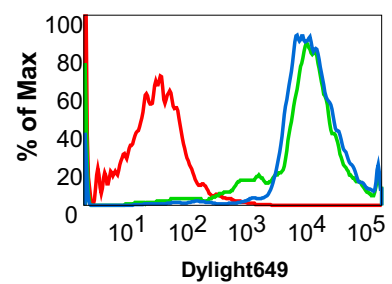

ScFv78-NP $\overline{\text { BSA-NP }}$ b

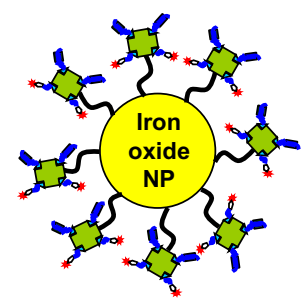

ScFv78-NP-BSA-Dylight649 (ScFv78-NP)

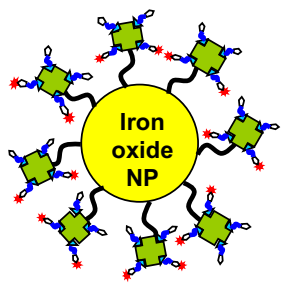

BSA-NP-BSA-Dylight649 (BSA-NP)

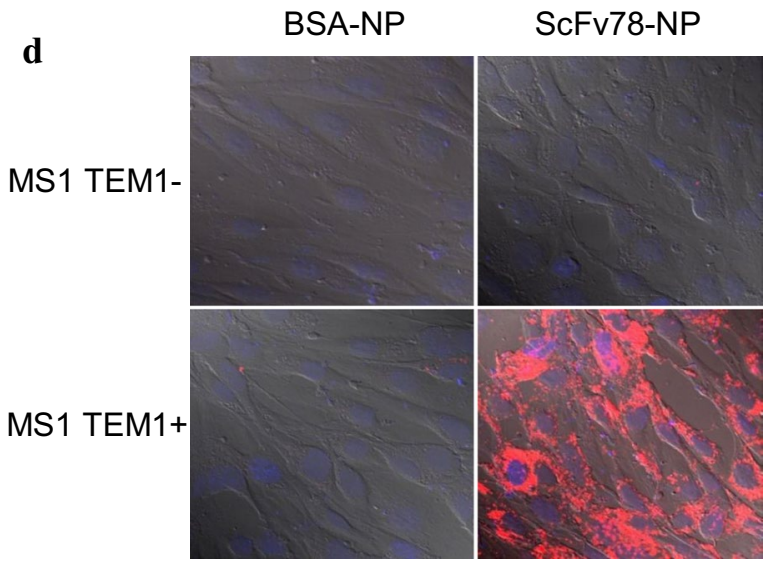

nanoparticles (red), BSA-NP(blue) and scFv78-NP(green). d scFv78NPor BSA-NP was incubated with MS1 and MS1-TEM1 (TEM1+) cells respectively in complete medium and then internalization was analyzed by confocal imaging

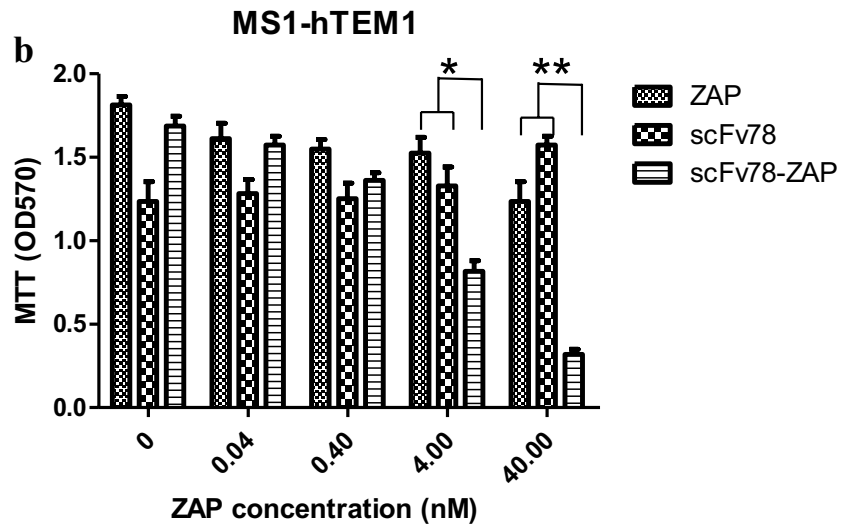

tive cells were incubated with serially diluted ZAP, scFv78 alone or scFv78-ZAP at the indicated concentrations. Then the viability was measured by MTT 96 h later. *p $<0.05 ; * * \mathrm{p}<0.01$

detection (detected more than 30 cycles) within the kidneys (Fig. 6). In contrast, we found high mTEM1 mRNA expression in TC1 tumors of all the animals (Fig. 6). There was no difference in mTEM1 expression observed between the two animal groups injected with scFv78 or control scFv (Fig. 6).

Fig. 4 TEM1-targeted drug delivery by scFv78. a Mouse endothelial cell line MS1 were incubated with serially diluted ZAP, scFv78 alone or scFv78-ZAP at the indicated concentrations. b MS1-hTEM1 posi-

excretion of scFv78-dye due to its small size [29-31]. To further confirm that this was non-specific accumulation, we ran quantitative PCR for TEM1 on liver and kidney biopsies taken from mice upon sacrifice. Murine TEM1 was undetectable in the liver and was found to be at the limit of 


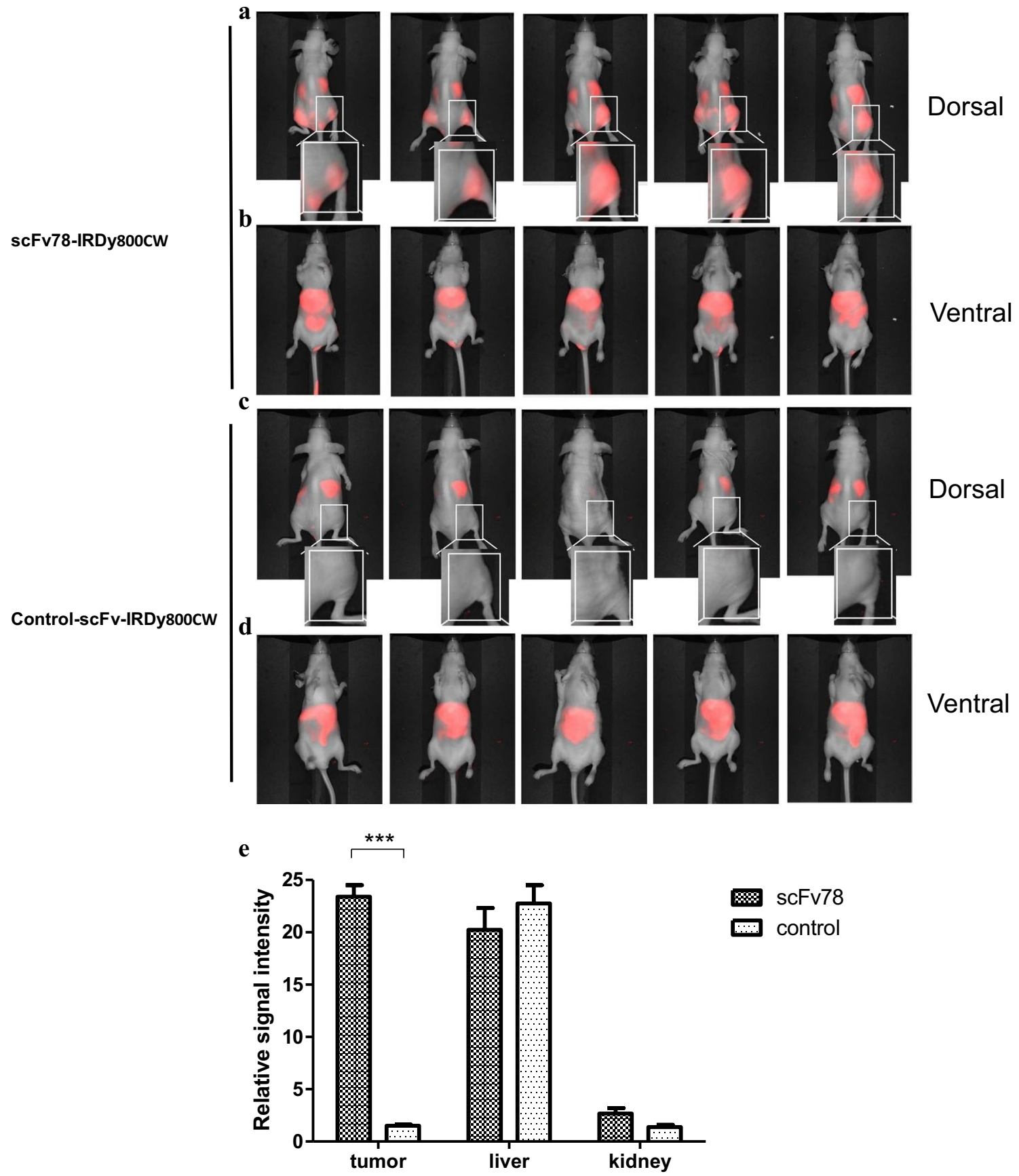

Fig. 5 In vivo tumor targeting by $\mathrm{scFv} 78$. This figure represents the biodistributions of $\operatorname{scFv} 78(\mathbf{a}, \mathbf{b})$ and control $\operatorname{scFv}(\mathbf{c}, \mathbf{d})$ in five animals $6 \mathrm{~h}$ post-injection in the ventral and dorsal positions. Magnified views of the framed tumor regions in two groups are shown in

\section{Discussion}

We previously developed the first fully human $\mathrm{scFv}$ against TEM1, an attractive vascular and stromal target. Here, we describe a number of potential uses for scFv78, including molecular imaging, immunotoxin-based therapy, and nanotherapy. This scFv was isolated from a human scFv library the inserts. Relative signal intensities of the tumor, liver and kidneys of both groups were plotted against the background intensity (e), $* * * \mathrm{p}<0.001$

with high affinity and specificity [21]. We have now shown that scFv78 has a low nanomolar binding affinity to its target in situ, consistent with most of the currently available monoclonal antibodies in the clinic [32, 33]. Importantly, we found that scFv78 binds to a conformational epitope and triggers rapid internalization of the naked $\mathrm{scFv}$ as well as immunoconjugates, highlighting its potential use for the 


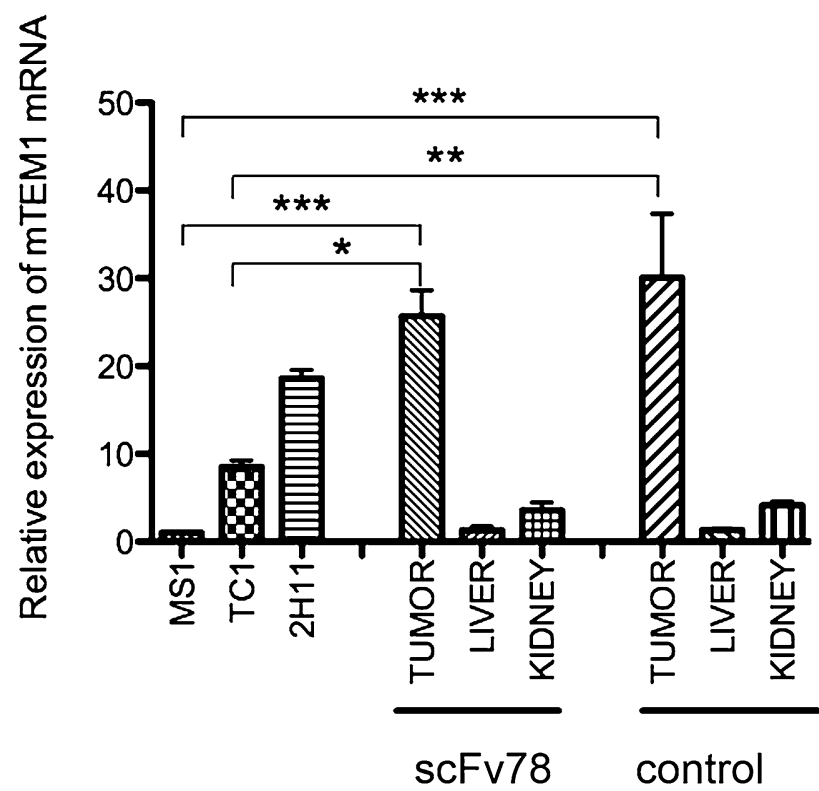

Fig. 6 Expression of mTEM1 in TC1 tumors by real-time quantitative PCR. TEM1 expression at the mRNA level in kidney, liver and tumor of the two groups. MS1 cells and 2H11 cells were used as negative and positive controls, respectively. The relative expression levels were plotted by $2^{\Delta \Delta \mathrm{CT}}$ using theMS1 level as $1 . * \mathrm{p}<0.05 ; * * \mathrm{p}<0.01$; $* * * \mathrm{p}<0.001$

delivery of immunotoxins or drug conjugates. Then, we demonstrated that scFv78 is cross-reactive with both human and murine TEM1. The cross-species reactivity of scFv78 is critically important for its clinical development, as it allows for the safety evaluation of TEM1-targeted therapy in mouse models, rendering its pathway towards the clinic more certain. Lastly, we have shown that scFv78 is able to efficiently target TEM1-positive tumors in vivo, as evidenced by our proof-of-principle optical imaging study.

Several recent clinical successes support the rationale for the clinical development of monoclonal antibodies as either naked or immunoconjugate therapeutics against cancer surface antigens. TEM1 is a promising therapeutic target. The tem $1^{-/-}$knockout mice, which have no outwardly detrimental phenotype, demonstrate dramatic reductions in tumor growth and metastasis [20]. Here we explore the utility of targeting TEM1 through scFv immunoconjugates, which could be used as imaging reagents, as well as delivery systems for therapeutic payloads. Internalization of any therapeutic cargo is required for its success. Data from our internalization assays demonstrated that both naked scFv78 as well as scFv78-functionalized NPs were rapidly internalized into TEM1-expressing cells. We believe this evidence provides sufficient rationale for the development of TEM1targeted immunotoxin and nanoparticle-based therapy using scFv78 as the delivery vehicle.
Antibody-drug conjugates (ADCs) are an emerging class of immunotoxins that offer a therapeutic approach specific for cancer cells expressing the targeted antigen [34, 35]. Due to their high potency, specificity, and low side effects, several ADCs are now in the advanced stage of clinical trials. As a proof of concept for the immunotoxin applications of scFv78, we conjugated scFv78 to saporin toxin. Saporinconjugated antibodies have been used in several clinical studies for tumor-targeted therapy [36, 37]. Although the immunogenicity of saporin renders it less amenable to repeated administrations in the clinic, it is suitable for developing proof-of-principle data. The in vitro data shown in this study clearly demonstrate that scFv78 can mediate the efficient delivery of saporin into TEM1-positive cells. The unique internalization property of scFv78 makes it a useful vehicle for the localized delivery of highly toxic agents specifically to tumor cells. The anti-TEM1 scFv78 characterized here may be reformatted to a full antibody format for conjugation to drugs or cytokines for the effective and preferential destruction of the tumor vasculature and stroma.

Our data also suggest that scFv78 could play a role in nanomedicine applications for tumor diagnosis and therapy $[38,39]$. In this study, we took advantage of the high affinity binding between streptavidin and biotin to label the NPs with scFv78 [40]. Considering the high immunogenicity of streptavidin, a better non-immunogenic coupling strategy should be developed to permit highly efficient conjugation without affecting the affinity of scFv78. The introduction of a free cysteine at the $\mathrm{C}$ terminus of $\mathrm{scFv} 78$ via a small flexible peptide linker might be a suitable strategy that would permit efficient site-specific conjugation through thiol-maleimide coupling [41, 42].

Specificity is a major challenge in the field of targeted macromolecules. To date, there are very few tumor-specific antigens that are entirely absent on normal tissues. Therefore, the safety of targeting a tumor marker with a novel antibody should be the first concern in translation. TEM1 is expressed by many types of solid tumors in both humans and mice, but is largely absent in normal mouse tissues [15]. Here, we provide the first in vivo imaging data of TEM1 expression in the mouse using scFv78, which is consistent with the previously established expression profile of TEM1 using in situ hybridization [15]. TEM1 is largely absent or present at negligible levels in normal mouse organs. In addition, our data agree with previous data presented by St. Croix [15]. We failed to detect TEM1 mRNA expression in the liver, where we had detected an accumulation of optical dye. This suggests that this was not targeted accumulation but was likely due to Kupffer cell pickup of the dye labeled antibodies. Altogether, the data of IHC confirm that scFv78 binds TEM1 in vivo, specifically at the vasculature site of the tumor and not at off-target organs, which 
provides encouragement for the further clinical development of scFv78.

The universally high expression of TEM1 in almost all types of solid tumors makes scFv78 an interesting tool for a variety of clinical applications, including molecular imaging. Although we used optical imaging here as a proof of principle, other imaging modalities with higher sensitivity, such as molecular positron emission tomography (PET), or a more anatomic definition, such as molecular magnetic resonance imaging (MRI), can be developed. Nevertheless, optical imaging has specific clinical applications, including intraoperative imaging with NIR fluorescence dyes that have deep penetrating properties in tissues (approximately $1 \mathrm{~cm})[43,44]$. Application of IRDye800CW-labeled scFv78 showed that it could be used as a tool in tumor imaging from the perspective of clinical application. Further safety evaluation of IRDye $800 \mathrm{CW}$ and other optimized dyes conjugation strategies will facilitate the translation of scFv78 for intraoperative optical imaging.

Tumor vasculature and stroma targeting represents an effective strategy for early tumor detection, diagnosis and treatment of many tumors. TEM1 is an example of one such attractive surface molecule. To the best of our knowledge, there is only one anti-TEM1 antibody presently under clinical development, MORAb004, which is a humanized antibody recognizing the fibronectin-binding domain of human TEM1. Unlike scFv78, however, MORAb004 does not cross-react with murine TEM1. ScFv78 is the first fully human $\mathrm{scFv}$ antibody in the field. Its unique properties, including rapid internalization and specificity for mouse and human TEM1, make it suitable for a variety of applications in tumor theranostics across a wide spectrum of solid tumors.

Acknowledgements This work was supported by NIH-Roadmap Transformative R01 CA156695 (George Coukos); the European Research Council advanced Grant 1400206AdG-322875 (George Coukos); the Claneil Foundation, Ivy Foundation, and Canary Foundation (George Coukos), the TAPITMAT Institute for Translational Medicine and Therapeutics (ITMAT) CA016520/NIH (Nathalie Scholler), the Honorable Tina Brozman Foundation (George Coukos, Aizhi Zhao), the National Cancer Institute FCCC-UPenn Cancer SPORE pilot Grant (Aizhi Zhao), and the National Science Foundation of China (30972788, Xiaopeng Yuan).

\section{Compliance with ethical standards}

Conflict of interest The authors have no potential conflicts of interest.

\section{References}

1. Folkman J (1971) Tumor angiogenesis: therapeutic implications. N Engl J Med 285(21):1182-1186. https://doi.org/10.1056/ NEJM197111182852108
2. Ausprunk DH, Folkman J (1977) Migration and proliferation of endothelial cells in preformed and newly formed blood vessels during tumor angiogenesis. Microvasc Res 14(1):53-65

3. Mueller MM, Fusenig NE (2004) Friends or foes - bipolar effects of the tumour stroma in cancer. Nat Rev Cancer 4(11):839-849. https://doi.org/10.1038/nrc1477

4. Motz GT, Coukos G (2011) The parallel lives of angiogenesis and immunosuppression: cancer and other tales. Nat Rev Immunol 11(10):702-711. https://doi.org/10.1038/nri3064

5. Ochsenbein AF, Klenerman P, Karrer U, Ludewig B, Pericin M, Hengartner H, Zinkernagel RM (1999) Immune surveillance against a solid tumor fails because of immunological ignorance. Proc Natl Acad Sci USA 96(5):2233-2238

6. Spiotto MT, Yu P, Rowley DA, Nishimura MI, Meredith SC, Gajewski TF, Fu YX, Schreiber H (2002) Increasing tumor antigen expression overcomes "ignorance" to solid tumors via crosspresentation by bone marrow-derived stromal cells. Immunity $17(6): 737-747$

7. Ashraf SQ, Umana P, Mossner E, Ntouroupi T, Brunker P, Schmidt C, Wilding JL, Mortensen NJ, Bodmer WF (2009) Humanised IgG1 antibody variants targeting membrane-bound carcinoembryonic antigen by antibody-dependent cellular cytotoxicity and phagocytosis. Br J Cancer 101(10):1758-1768. https:// doi.org/10.1038/sj.bjc.6605355

8. McLaughlin P, Grillo-Lopez AJ, Link BK, Levy R, Czuczman MS, Williams ME, Heyman MR, Bence-Bruckler I, White CA, Cabanillas F, Jain V, Ho AD, Lister J, Wey K, Shen D, Dallaire BK (1998) Rituximab chimeric anti-CD20 monoclonal antibody therapy for relapsed indolent lymphoma: half of patients respond to a four-dose treatment program. J Clin Oncol 16(8):2825-2833

9. Bagley RG, Rouleau C, St Martin T, Boutin P, Weber W, Ruzek M, Honma N, Nacht M, Shankara S, Kataoka S, Ishida I, Roberts BL, Teicher BA (2008) Human endothelial precursor cells express tumor endothelial marker 1/endosialin/CD248. Mol Cancer Ther 7(8):2536-2546. https://doi.org/10.1158/1535-7163. MCT-08-0050

10. Simonavicius N, Robertson D, Bax DA, Jones C, Huijbers IJ, Isacke CM (2008) Endosialin (CD248) is a marker of tumor-associated pericytes in high-grade glioma. Mod Pathol 21(3):308-315. https://doi.org/10.1038/modpathol.3801006

11. Wesseling P, Schlingemann RO, Rietveld FJ, Link M, Burger PC, Ruiter DJ (1995) Early and extensive contribution of pericytes/ vascular smooth muscle cells to microvascular proliferation in glioblastoma multiforme: an immuno-light and immuno-electron microscopic study. J Neuropathol Exp Neurol 54(3):304-310

12. MacFadyen JR, Haworth O, Roberston D, Hardie D, Webster MT, Morris HR, Panico M, Sutton-Smith M, Dell A, van der Geer P, Wienke D, Buckley CD, Isacke CM (2005) Endosialin (TEM1, CD248) is a marker of stromal fibroblasts and is not selectively expressed on tumour endothelium. FEBS Lett 579(12):25692575. https://doi.org/10.1016/j.febslet.2005.03.071

13. Christian S, Ahorn H, Koehler A, Eisenhaber F, Rodi HP, GarinChesa P, Park JE, Rettig WJ, Lenter MC (2001) Molecular cloning and characterization of endosialin, a C-type lectin-like cell surface receptor of tumor endothelium. J Biol Chem 276(10):7408-7414. https://doi.org/10.1074/jbc.M009604200

14. Rettig WJ, Garin-Chesa P, Healey JH, Su SL, Jaffe EA, Old LJ (1992) Identification of endosialin, a cell surface glycoprotein of vascular endothelial cells in human cancer. Proc Natl Acad Sci USA 89(22):10832-10836

15. Carson-Walter EB, Watkins DN, Nanda A, Vogelstein B, Kinzler KW, St Croix B (2001) Cell surface tumor endothelial markers are conserved in mice and humans. Cancer Res 61(18):6649-6655

16. Becker R, Lenter MC, Vollkommer T, Boos AM, Pfaff D, Augustin HG, Christian S (2008) Tumor stroma marker endosialin (Tem1) is a binding partner of metastasis-related protein Mac-2 
BP/90K. FASEB J 22(8):3059-3067. https://doi.org/10.1096/ fj. $07-101386$

17. Tomkowicz B, Rybinski K, Foley B, Ebel W, Kline B, Routhier E, Sass P, Nicolaides NC, Grasso L, Zhou Y (2007) Interaction of endosialin/TEM1 with extracellular matrix proteins mediates cell adhesion and migration. Proc Natl Acad Sci USA 104(46):1796517970. https://doi.org/10.1073/pnas.0705647104

18. Christian S, Winkler R, Helfrich I, Boos AM, Besemfelder E, Schadendorf D, Augustin HG (2008) Endosialin (Tem1) is a marker of tumor-associated myofibroblasts and tumor vesselassociated mural cells. Am J Pathol 172(2):486-494. https://doi. org/10.2353/ajpath.2008.070623

19. Davies G, Cunnick GH, Mansel RE, Mason MD, Jiang WG (2004) Levels of expression of endothelial markers specific to tumourassociated endothelial cells and their correlation with prognosis in patients with breast cancer. Clin Exp Metastasis 21(1):31-37

20. Nanda A, Karim B, Peng Z, Liu G, Qiu W, Gan C, Vogelstein B, St Croix B, Kinzler KW, Huso DL (2006) Tumor endothelial marker 1 (Tem1) functions in the growth and progression of abdominal tumors. Proc Natl Acad Sci USA 103(9):3351-3356. https://doi.org/10.1073/pnas.0511306103

21. Zhao A, Nunez-Cruz S, Li C, Coukos G, Siegel DL, Scholler N (2011) Rapid isolation of high-affinity human antibodies against the tumor vascular marker Endosialin/TEM1, using a paired yeast-display/secretory scFv library platform. J Immunol Methods 363(2):221-232. https://doi.org/10.1016/j.jim.2010.09.001

22. Scholler N, Garvik B, Quarles T, Jiang S, Urban N (2006) Method for generation of in vivo biotinylated recombinant antibodies by yeast mating. J Immunol Methods 317(1-2):132-143. https://doi. org/10.1016/j.jim.2006.10.003

23. Berman HM, Westbrook J, Feng Z, Gilliland G, Bhat TN, Weissig H, Shindyalov IN, Bourne PE (2000) The protein data bank. Nucleic Acids Res 28(1):235-242

24. Pearson WR (1990) Rapid and sensitive sequence comparison with FASTP and FASTA. Methods Enzymol 183:63-98

25. Jones DT, Taylor WR, Thornton JM (1992) A new approach to protein fold recognition. Nature 358(6381):86-89. https://doi. org $/ 10.1038 / 358086 \mathrm{a} 0$

26. Chen R, Li L, Weng Z (2003) ZDOCK: an initial-stage proteindocking algorithm. Proteins 52(1):80-87. https://doi.org/10.1002/ prot. 10389

27. Prantner AM, Nguyen CV, Scholler N (2013) Facile immunotargeting of nanoparticles against tumor antigens using sitespecific biotinylated antibody fragments. J Biomed Nanotechnol 9(10):1686-1697

28. Rabasa Capote A, González JE, Rodríguez-Vera L, López A, Sánchez Ramírez B, Garrido Hidalgo G (2012) Pharmacokinetics and biodistribution study of 7A7 anti-mouse epidermal growth factor receptor monoclonal antibody and its $\mathrm{F}(\mathrm{ab}$ ')(2) fragment in an immunocompetent mouse model. ISRN Pharmacol 2012:417515. https://doi.org/10.5402/2012/417515

29. Crombet T, Torres L, Neninger E, Catala M, Solano ME, Perera A, Torres O, Iznaga N, Torres F, Perez R, Lage A (2003) Pharmacological evaluation of humanized anti-epidermal growth factor receptor, monoclonal antibody $\mathrm{h}-\mathrm{R} 3$, in patients with advanced epithelial-derived cancer. J Immunother 26(2):139-148

30. Lewis MR, Boswell CA, Laforest R, Buettner TL, Ye D, Connett JM, Anderson CJ (2001) Conjugation of monoclonal antibodies with TETA using activated esters: biological comparison of $64 \mathrm{Cu}-\mathrm{TETA}-1 \mathrm{~A} 3$ with $64 \mathrm{Cu}-\mathrm{BAT}-2 \mathrm{IT}-$ 1A3. Cancer Biother Radiopharm 16(6):483-494. https://doi. org/10.1089/10849780152752083

31. Behr TM, Sharkey RM, Juweid ME, Blumenthal RD, Dunn RM, Griffiths GL, Bair HJ, Wolf FG, Becker WS, Goldenberg DM
(1995) Reduction of the renal uptake of radiolabeled monoclonal antibody fragments by cationic amino acids and their derivatives. Cancer Res 55(17):3825-3834

32. Liang WC, Wu X, Peale FV, Lee CV, Meng YG, Gutierrez J, Fu L, Malik AK, Gerber HP, Ferrara N, Fuh G (2006) Cross-species vascular endothelial growth factor (VEGF)-blocking antibodies completely inhibit the growth of human tumor xenografts and measure the contribution of stromal VEGF. J Biol Chem 281(2):951-961. https://doi.org/10.1074/jbc.M508199200

33. Troise F, Cafaro V, Giancola C, D'Alessio G, De Lorenzo C (2008) Differential binding of human immunoagents and herceptin to the ErbB2 receptor. FEBS J 275(20):4967-4979. https://doi. org/10.1111/j.1742-4658.2008.06625.x

34. Krop I, Winer EP (2014) Trastuzumab emtansine: a novel antibody-drug conjugate for HER2-positive breast cancer. Clin Cancer Res 20(1):15-20. https://doi.org/10.1158/1078-0432. CCR-13-0541

35. Okeley NM, Miyamoto JB, Zhang X, Sanderson RJ, Benjamin DR, Sievers EL, Senter PD, Alley SC (2010) Intracellular activation of SGN-35, a potent anti-CD30 antibody-drug conjugate. Clin Cancer Res 16(3):888-897. https://doi.org/10.1158/1078-0432. CCR-09-2069

36. Bonardi MA, Bell A, French RR, Gromo G, Hamblin T, Modena D, Tutt AL, Glennie MJ (1992) Initial experience in treating human lymphoma with a combination of bispecific antibody and saporin. Int J Cancer Suppl 7:73-77

37. French RR, Bell AJ, Hamblin TJ, Tutt AL, Glennie MJ (1996) Response of B-cell lymphoma to a combination of bispecific antibodies and saporin. Leuk Res 20(7):607-617

38. Singh A, Sahoo SK (2014) Magnetic nanoparticles: a novel platform for cancer theranostics. Drug Discov Today 19(4):474-481. https://doi.org/10.1016/j.drudis.2013.10.005

39. Howell M, Wang C, Mahmoud A, Hellermann G, Mohapatra SS, Mohapatra S (2013) Dual-function theranostic nanoparticles for drug delivery and medical imaging contrast: perspectives and challenges for use in lung diseases. Drug Deliv Transl Res 3(4):352-363. https://doi.org/10.1007/s13346-013-0132-4

40. Chilkoti A, Tan PH, Stayton PS (1995) Site-directed mutagenesis studies of the high-affinity streptavidin-biotin complex: contributions of tryptophan residues 79, 108, and 120. Proc Natl Acad Sci USA 92(5):1754-1758

41. Nellis DF, Ekstrom DL, Kirpotin DB, Zhu J, Andersson R, Broadt TL, Ouellette TF, Perkins SC, Roach JM, Drummond DC, Hong K, Marks JD, Park JW, Giardina SL (2005) Preclinical manufacture of an anti-HER2 scFv-PEG-DSPE, liposome-inserting conjugate. 1. Gram-scale production and purification. Biotechnol Prog 21(1):205-220. https://doi.org/10.1021/bp049840y

42. Figini M, Martin F, Ferri R, Luison E, Ripamonti E, Zacchetti A, Mortarino M, Di Cioccio V, Maurizi G, Allegretti M, Canevari S (2009) Conversion of murine antibodies to human antibodies and their optimization for ovarian cancer therapy targeted to the folate receptor. Cancer Immunol Immunother 58(4):531-546. https:// doi.org/10.1007/s00262-008-0575-5

43. Ogawa M, Kosaka N, Choyke PL, Kobayashi H (2009) In vivo molecular imaging of cancer with a quenching near-infrared fluorescent probe using conjugates of monoclonal antibodies and indocyanine green. Cancer Res 69(4):1268-1272. https://doi. org/10.1158/0008-5472.CAN-08-3116

44. Nakajima T, Mitsunaga M, Bander NH, Heston WD, Choyke PL, Kobayashi H (2011) Targeted, activatable, in vivo fluorescence imaging of prostate-specific membrane antigen (PSMA) positive tumors using the quenched humanized J591 antibody-indocyanine green (ICG) conjugate. Bioconjug Chem 22(8):1700-1705. https://doi.org/10.1021/bc2002715 